\title{
¿Pedagogía activa o métodos activos? El caso del aprendizaje activo en la universidad
}

\author{
Active Pedagogy or Active Methods? The Case of Active Learning at University \\ Pedagogia ativa ou métodos ativos? 0 caso da aprendizagem ativa na universidade
}

Roberto Espejo Leupín *

Unidad de Desarrollo Docente, Dirección de Calidad Educativa, Vicerrectoría Académica Universidad Central de Chile, Santiago, Chile
Recibido: 08.01.16

Aceptado: 25.04.16
RESUMEN. En este artículo discutimos el enfoque del aprendizaje activo que se ha difundido en las aulas universitarias, relacionándolo con sus antecesores pedagógicos, específicamente el movimiento de la Escuela Nueva Europea y la Educación Progresista Norteamericana. Esta relación nos permite entender en una perspectiva histórica el abanico de métodos de enseñanza-aprendizaje que se están utilizando en la Universidad, incluyendo diferencias con sus antecesores. En particular, discutimos la diferencia entre métodos activos y la noción más general de pedagogía activa, la que implica una filosofía de la educación que se centra de manera más radical en el estudiante. Esto último nos permite esbozar posibles líneas de evolución de la práctica de la pedagogía activa en la Universidad.
Palabras claves: pedagogía, aprendizaje activo, historia de la educación.

ABSTRACT. In this paper we discuss the active learning approach that has been spread in university classrooms in relation to its pedagogical predecessors, particularly the New European School movement and the American Progressive Education. This relationship allows us to understand the array of teaching-learning methods used today at university under a historical perspective, including differences with its predecessors. Particularly, we will discuss the difference between active methods and the more general concept of active pedagogy, which implies an educational philosophy more radically centered on the students. This enables us to outline possible evolution lines in the practice of active pedagogy at university. 
RESUMO. Neste artigo discutimos a abordagem da aprendizagem ativa que foi difundida nas salas de aulas universitárias relacionando-a com seus antecessores pedagógicos, particularmente o movimento da Escola Nova Europeia e a Educação Progressiva Norteamericana. Esta relação nos permite entender em uma perspectiva histórica a gama de métodos de ensino-aprendizagem utilizados hoje em dia na Universidade, incluindo diferenças com seus antecessores. Em particular, discutimos a diferença entre métodos ativos e a noção mais geral de pedagogia ativa, o que implica em uma filosofia da educação que se concentra de maneira mais radical no estudante. Este último ponto nos permite esboçar possíveis linhas de evolução da prática da pedagogia ativa na Universidade.

La discusión sobre los métodos pedagógicos utilizados por los docentes en las aulas universitarias ha tomado un lugar importante en los últimos años. En especial, los denominados métodos activos, es decir, aquellos que promueven la actividad de los estudiantes en clase, en contraposición a una actitud pasiva, aparecen formando algo así como el discurso hegemónico en casi todas las instituciones de Educación Superior. Esto se ha comprobado -como se indica más adelante en el texto- en investigaciones al respecto de que el aprendizaje de los estudiantes se beneficia de la utilización de dichos métodos, por lo que todo profesor universitario debería considerar esto a la hora de preparar y llevar a cabo sus clases. Sin embargo, en estricto rigor, el uso de estos métodos no es una idea nueva en el ámbito educativo. Una larga tradición nos habla del aprendizaje centrado en la actividad del estudiante. Desde Rousseau hasta Dewey, pasando por pedagogos como Pestalozzi y Froebel, se ha insistido en la importancia de la relación entre la actividad del estudiante y el aprendizaje de un cierto tema. La universidad pareciera en la actualidad ser heredera de esta larga tradición pedagógica, aunque muchos de los métodos que se promueven hoy son presentados muchas veces como innovaciones desconectadas de esta historia.

Así, la hipótesis central de este trabajo es que podemos encontrar antecedentes del aprendizaje activo en la universidad en los movimientos escolares que enfatizaron la centralidad del estudiante y el impacto que tiene el "hacer" en su aprendizaje, y que podemos encontrar en estos movimientos una experiencia valiosa que -en principio- puede entregar elementos relevantes para orientar el desarrollo de los métodos activos en el ámbito universitario.

El examen histórico del origen de la pedagogía activa se hace entonces esencial. Para esto tomaremos como referencia una parte de la historia de esta idea, los movimientos de la Escuela Nueva Europea y a la Pedagogía Progresista en los Estados Unidos, así como a algunos de sus epígonos que han llegado hasta nuestros días.

\section{SOBRE EL APRENDIZAJE ACTIVO EN LA UNIVERSIDAD}

Con relación a la pedagogía universitaria, Béchard (2008) ha realizado la caracterización del núcleo referencial del espacio intelectual de esta disciplina mediante el análisis de las referencias bibliográficas más citadas en tres revistas representativas. A partir de estas referencias, Béchard identifica tres programas de investigación (en el sentido de Lakatos): al el programa centrado en el desarrollo de las personas, b) el centrado en la pedagogía de las competencias y c) el centrado en el cambio pedagógico (Béchard, 2008, p. 561). Principalmente en el segundo y tercer ámbito encontramos la problemática de los procesos de enseñanza-aprendizaje y de los modelos pedagógicos innovadores. Con relación a esto, De Ketele (2010) subraya la predominancia de la discusión de actividades de aprendizaje en las publicaciones del campo. En 
otras palabras, el análisis del impacto en los procesos de los estudiantes de distintas metodologías de enseñanza-aprendizaje, donde se incluye la utilización de los denominados métodos activos. Este énfasis se encuentra en la línea de lo que ha sido denominado un paradigma centrado en el aprendizaje (Barr \& Tagg, 1995), por lo que es normal encontrar la expresión aprendizaje activo.

Atendiendo a la heterogeneidad conceptual de esta idea, el informe de la Asociación para el estudio de la educación superior (Bonwell \& Eison, 1991) ya señalaba que este término (active learning) no ha sido jamás definido de forma precisa en la literatura. Sin embargo existirían ciertas características asociadas a la utilización de estrategias que lo promueven en clases, a saber: al los estudiantes hacen más que solo escuchar, b) la transmisión de información se enfatiza menos y se da espacio para el desarrollo de las capacidades de los estudiantes, cl los estudiantes se implican en un proceso de pensamiento de orden superior lanálisis, síntesis, evaluación, siguiendo a Bloom), d) los estudiantes se implican en actividades (por ejemplo la lectura, la escritura o el debate) y e) se enfatiza la exploración de actitudes y valores de los estudiantes. Así, a partir de estas características, los autores proponen una definición de trabajo: el aprendizaje activo sería un aprendizaje "que implica a los estudiantes en el hacer y en la reflexión sobre lo que están haciendo" (Bonwell \& Eison, 1990, p. 19).

Algunos antecedentes del aprendizaje activo. El desarrollo de la idea del aprendizaje activo en la Escuela es una historia apasionante. Debemos considerar que no se trata de un fenómeno homogéneo (Ohayon, Ottavi \& Savoye, 2004; Resweber, 1986), sino que es más bien una corriente donde se encuentran muchas tendencias, en función de los países y pedagogos implicados. Podemos distinguir inicialmente el movimiento de la Educación Nueva en Europa lla Escuela Nueval y su versión en los Estados Unidos, la Educación Progresista. La relación entre el movimiento americano y el europeo es un proceso de influencias recíprocas, como ha sido subrayado por algunos investigadores (Luzuriaga, 1944; Oelkers, 2006).

Lorenzo Luzuriaga (1944) propone distinguir cuatro períodos principales en el desarrollo de estas ideas. El primero se caracteriza por la creación de las primeras Escuelas Nuevas en Europa y América (1889-1900), donde encontramos la Escuela de Abbotsholme (1889) y la Escuela de Bedales (1893) en Inglaterra y las Escuelas del Campo del Dr. Lietz (1898) en Alemania, la Escuela des Roches (1899) de E. Demolins en Francia y la Escuela Primaria Universitaria de Chicago (1896) creada por John Dewey. Este período estaría compuesto de iniciativas concretas y no de especulaciones teóricas. En el segundo período se hace la formulación teórica de las nuevas ideas pedagógicas (1900-1907), por ejemplo a través de los escritos de Dewey en Estados Unidos y de Georg Kerschensteiner en Alemania. El tercer período corresponde a la creación y publicación de los primeros métodos activos (1907-1918): el método Montessori en Roma, el Decroly en Bruselas, el Plan Dalton, el método de proyectos de Kilpatrick, etc. La cuarta etapa se caracteriza por la difusión, consolidación y oficialización de las ideas y de los métodos de la nueva educación (a partir de 1918): la creación de la Progressive education association (1919) en Estados Unidos y de la Ligue internationale pour l'éducation nouvelle (1921) en Calais (Luzuriaga, 1944).

Se podría hablar de los antecedentes históricos de la Educación Nueva a partir de algunos movimientos pedagógicos que se desarrollaron en el Renacimiento, particularmente ligados al movimiento humanista. Se opone así a la educación medieval dogmática y autoritaria una educación más crítica y libre que pone la transmisión de las verdades de la teología y la escolástica frente a las investigaciones de la filosofía y de la ciencia. Los nombres de Vitorino da Feltre, de Erasmo, Vives, Rabelais y Montaigne son ejemplos de esta conciencia, anunciadora de la educación nueva.

Para Adolphe Ferrière, difusor de estas ideas, lo que es esencial en este movimiento pedagógico es la importancia de la espontaneidad y la expresión creativa del niño (Ferrière, 2004). Para esto es necesario considerar todas sus dimensiones: la intelectual, la afectiva y la volitiva: "la razón no lo es todo. Al lado de la inteligencia y de la intuición está el hombre, el sentimiento; está la voluntad, la vida bajo sus múltiples aspectos" (Ferrière, 2004, p. 52). Por ejemplo, en uno de sus artículos de la Revista Ilustrada de París, Ferrière, haciendo un reporte de las escuelas nuevas 
en Alemania, Suiza, Inglaterra y Francia, señala como características centrales de estas: al la importancia de la educación física, b) la educación de la razón y cl la educación moral (Ferrière, 1911). Este discurso sobre el desarrollo integral del niño no es nuevo: la historia de las ideas pedagógicas nos muestra a precursores tales como Rousseau, Pestalozzi y Froebel. De hecho, Ferrière los reconoce como antecedentes pero -y esto es un punto importante- los clasifica como "genios intuitivos" (Ferrière, 1911, p. 620; 2004, p.37). Para él la diferencia entre el aporte de estos pedagogos y el de la Educación Nueva es el fundamento científico, donde la idea del desarrollo psicológico del niño da el fundamento a la actividad:

“... la Escuela Activa es ante todo, de forma general, la aplicación de las leyes de la psicología a la educación de los niños. La sociología, por una parte, y por otra la psicología genética que estudia el desarrollo de los seres, son las ciencias madres de esta ciencia aplicada o de este arte que es la educación" (Ferrière, 2004, p.231).

Un elemento importante de las ideas de Ferrière es la diferencia que establece entre la escuela activa les decir, la educación nueval y los llamados métodos activos. Para él, estos últimos tienen como fin la materia de los exámenes y no necesariamente el desarrollo del niño.

Llamar escuela activa a los métodos activos, compromiso nefasto con las exigencias de leyes escolares absurdas, he aquí el peligro, la trampa en la que el gusto por el mínimo esfuerzo hace caer a numerosos institutores todos los días. Los métodos activos son un procedimiento más entre muchos otros, para hacer asimilar a los alumnos un programa fijo de antemano (Ferrière, 2004, p. 49).

Pareciera ser que para Ferrière el núcleo de la escuela activa, la educación como acompañamiento de la evolución creativa del niño, no se debe confundir con los medios, como es el caso de los métodos activos. Así, critica Ferrière el Plan Dalton y subraya la potencial perversión del método Decroly llos centros de interés) si la motivación no viene verdaderamente de los niños. Sin embargo, esta crítica deber ser tomada con cuidado, ya que algunos investigadores consideran la utilización de los métodos activos como uno de los criterios más seguros de la implementación de las ideas de la Educación Nueva (Hameline, 2006).

Luego de la Segunda Guerra Mundial, los congresos de la Liga internacional por la educación nueva terminaron y el movimiento desapareció en esa forma. Sin embargo, sus ideas se perpetuaron en movimientos nacionales en algunos países como Francia y Bélgica (Resweber, 1986, p. 119), el movimiento Freinet, y otras escuelas puntuales (Resweber, 1986; Vasquez \& Oury, 1967).

El otro punto de referencia fundamental es el movimiento de la Educación Progresista en Estados Unidos, en particular el trabajo de John Dewey y su conocido concepto del "aprender a través del hacer" (learning by doing). Sin embargo, no se debe pensar que Dewey fue el único en su época en comprometerse en un movimiento de reforma de la Escuela (Deledalle, 1995), aunque por su inmensa producción escrita, su trabajo en la Escuela Experimental de la Universidad de Chicago -donde trabajó con su esposa-y sus ideas innovadoras se le reconoce como el representante por excelencia del movimiento progresista.

En Dewey, la importancia de la actividad se explica por el lugar central que da a la experiencia en su teoría de la educación. Según él, la biología nos enseña que "allí donde está la vida está la actividad", y esta actividad debe ser continua y ajustada al entorno. Se trata de un ajuste adaptativo que no puede ser completamente pasivo (Dewey, 2003, p. 91). Esta observación biológica permite a Dewey elaborar una teoría de la experiencia como un "hacer" que actúa sobre el entorno de un organismo. Por otra parte, los cambios producidos por el ambiente actúan sobre el mismo organismo y sus actividades. La actividad, a partir de los intereses del niño, es vista entonces como central (Dewey, 1900, p. 38).

Otro personaje importante por la difusión del movimiento progresista fue William Kilpatrick (18711965). Más que un teórico, fue un personaje que aplicó de manera remarcable las ideas de Dewey, bajo la forma del "método por proyectos", implementando un dispositivo pedagógico que subrayaba la centralidad del interés del niño (Zilversmit, 1993). En efecto, como ha sido señalado por María del Pozo Andrés (2009b), los nuevos métodos de enseñanza que incorporaban y armonizaban los elementos didácticos como la 
motivación y la socialización eran vistos como una de las características centrales de la Educación Nueva en Estados Unidos. El método por proyectos de Kilpatrick integraba estos dos conceptos y los aplicaba.

Incluso si las ideas de la Educación Progresista fueron implementadas heterogéneamente según los pedagogos y sus situaciones individuales, Zilversmit (1993) identifica un núcleo de tres características del movimiento, en el que vemos reflejadas las ideas de Ferrière que hemos discutido más arriba:

(a) Los progresistas consideraban que una escuela progresista seguía un curriculum centrado en el niño y no centrado en el tema de estudios. La escuela debía aprovechar el deseo natural de aprender del niño.

(b) La escuela debía desarrollar al niño en su totalidad, es decir, promover su desarrollo emocional, físico e intelectual. Detrás de ese punto existe una discusión sobre el equilibrio ideal entre estos aspectos, sobre todo en relación con el programa escolar y la importancia dada a los distintos temas de estudio.

(c) Los progresistas consideraban que el niño debía tener un rol activo en la determinación de los contenidos de su aprendizaje. Este punto esconde, evidentemente, una amplia discusión de la responsabilidad de los niños con relación a esto.

Luego de la Segunda Guerra Mundial, el discurso educativo se enfocó en los aprendizajes llamados fundamentales (lectura, escritura) y las ideas del movimiento progresista fueron hasta cierto punto rechazadas. El éxito soviético del Sputnik, en 1957, mostró a la sociedad norteamericana la diferencia entre su sistema educativo y el soviético (Herold, 1974). Buscando a responsables, las ideas pedagógicas de Dewey recibieron fuertes críticas (Zilversmith, 1993). En los años 60 y 70 se observa la reaparición de algunas de estas ideas en los trabajos de Paul Goodman, Jonathan Kozol y Carl Rogers, así como en el movimiento de las Free Schools (Miller, 2002). Se aprecia cómo a pesar de los cambios de política educativa, parecería que las ideas del movimiento progresista permanecieron siempre en algunos círculos como una influencia importante.
El desarrollo de la dinámica de grupos a partir de los trabajos de Kurt Lewin en los años 40 es también relevante para entender la continuidad de la Educación Progresista. La influencia de Carl Rogers, padre de la psicología humanista, estuvo también muy presente a partir de los años 60. Nociones como la educación centrada en la persona, que promueve el desarrollo completo de esta, es decir, no tan solo en el aspecto intelectual sino en el afectivo, nos recuerda el discurso de la Escuela Activa. De la misma manera, la idea de la actividad y del compromiso del aprendiz son esenciales, entendiendo que el único aprendizaje que influencia realmente el comportamiento de un individuo es el que descubre por sí mismo y del cual se apropia (Rogers \& Freiberg, 1994). Vemos una continuación de las ideas de Dewey sobre la actividad a través de la mirada psicológica de Rogers y la vuelta sobre el campo educativo: de hecho Rogers tomó clases con Kilpatrick en el Teachers College, de donde podemos colegir la influencia de Dewey sobre él.

\section{El origen del discurso sobre el aprendizaje activo en} la universidad. Es difícil trazar de manera precisa el momento de la aplicación de los métodos activos en la enseñanza superior. Lo más probable es que siempre hayan existido profesores universitarios excepcionales que han utilizado dichas estrategias pedagógicas en sus clases, incluso de manera intuitiva y sin acercarse ex profeso a un movimiento pedagógico. Sin embargo, si intentamos rastrear los antecedentes del discurso, los encontramos en un inicio en los Estados Unidos, muy probablemente asociados a la influencia del movimiento de la Educación Progresista, en particular a los escritos de Dewey. Así, es muy interesante remarcar la existencia de algunas iniciativas universitarias experimentales, incluso al inicio del siglo $X X$, las cuales han intentado utilizar las ideas de la Educación Progresista (Reynolds, 1997; Townsend, Jackson \& Wiese, 1992).

En efecto, las investigaciones sobre el método pedagógico en la enseñanza superior en los Estados Unidos datan de los años 20. Por ejemplo, Frank Costin (1972) hace una revisión de las investigaciones sobre la pregunta de la eficacia de los cursos magistrales con relación a otros métodos de enseñanza, tales como la discusión en clases lla primera investigación 
data de 1925), la utilización de proyectos centrados en el estudiante (la primera investigación data de 1950), y la lectura individual y la autoinstrucción (primera investigación en 1928).

Sin embargo, estas investigaciones no permiten deducir una superioridad consistente de los métodos activos por sobre las clases magistrales (Costin, 1972). McKeachie (1990) ha mostrado también que las investigaciones en educación superior relativas a la utilización de los métodos más activos datan de los años 20 para el caso de la comparación entre cursos magistrales y la discusión. Así mismo, traza la influencia de la psicología de grupos de Lewin y la no directividad de Rogers en los métodos activos. Sobre el grupo, se nota la emergencia de investigaciones en los años 30 y 40 (McKeachie, 1990). Así, la pregunta de la posibilidad de utilizar métodos activos en la enseñanza universitaria no es algo nuevo y ha estado ligado, al menos en los Estados-Unidos, a la eficacia de la enseñanza (McKeachie, Pintrinch, Lin \& Smith, 1987) y a una metodología de investigación empírica. La existencia de estas investigaciones genera entonces las condiciones propicias para la emergencia de un discurso oficial sobre la utilización de estos métodos.

En Estados Unidos parecería que el discurso generalizado sobre la importancia de los metodos activos data de los años 80 y aparece oficialmente con el informe Involvement in Learning: Realizing the Potential of American Higher Education presentado al Secretario de Educación. Este documento fue pedido a un grupo multidisciplinario de expertos con el fin de establecer condiciones de excelencia para la educación superior americana en vista de mejorarla (National Institute of Education, 1984).

La segunda recomendación del documento establece que los profesores universitarios "deberían utilizar más los modos de enseñanza activos y pedir a los estudiantes que se responsabilicen de su aprendizaje" (National Institute of Education, 1984, p. 33). De la misma manera, la tercera recomendación subraya la utilización de las herramientas tecnológicas, en particular las computadoras. Este documento es importante, ya que marcará la política educativa en relación con los métodos pedagógicos promovidos en la enseñanza superior. En la misma dirección, nos parece fundamental el artículo publicado por A. Chikering y Z. Gamson en 1987 donde proponen siete principios para una buena práctica de enseñanza universitaria, los cuales -según los autores- estaban basados en investigaciones realizadas desde los años 30. Entre esos principios aparece la importancia clara de promover el aprendizaje activo de los estudiantes (Chickering \& Gamson, 1987).

En 1991, Charles Bonwell y James Eison publicaron el informe Active learning: creating excitement in the classroom, presentado a la Asociación para el estudio de la Educación Superior. En este documento se discuten varios métodos de aprendizaje activo, enfatizando los autores la importancia de los esfuerzos realizados por los profesores universitarios en su implementación. Al mismo tiempo señalan la necesidad de fundamentarlos científicamente a través de investigaciones cuantitativas y cualitativas actualizadas (Bonwell \& Eison, 1991, vii).

En Europa los acontecimientos de la revuelta estudiantil de 1968 provocaron la implementación de modelos universitarios donde los métodos activos tuvieron una importancia mayor. Los casos de las universidades de Roskilde y de Aalborg en Dinamarca y de la Universidad de Paris 8-Vincennes en Francia son ejemplos de espacios donde los profesores pudieron experimentar con un currículum multidisciplinario y con métodos activos. En la Universidad de Paris 8, los dispositivos pedagógicos intentan romper con los cursos magistrales y la relación pedagógica autoritaria e "imaginar un nuevo tipo de funcionamiento donde el profesor ya no es la única referencia y donde los estudiantes quieren hacerse cargo de su aprendizaje" (Blondeau \& Couëdel, 2002).

La creación del Espacio Europeo de Educación Superior ha dado lugar a recomendaciones con relación a la utilización de métodos activos en la universidad (Padilla y Gil, 2008). Sin embargo, como lo muestra María Del Pozo Andrés, los documentos originales del Proceso de Bolonia (comenzado en 1999) no tocan directamente los métodos por promover en los procesos de enseñanza-aprendizaje. Se debió esperar hasta el 2007 para tener referencias explícitas respecto de este 
punto, y parece ser que se trata de un tema que solo un grupo restringido de países europeos consideran en sus informes, como por ejemplo España, Dinamarca y Finlandia (Del Pozo Andrés, 2009a).

\section{MÉTODOS ACTIVOS: LO QUE SE HACE AHORA EN LA UNIVERSIDAD.}

Como se mencionó anteriormente, el movimiento de la Escuela Activa señala como central el aporte de la psicología en el ámbito educativo. En el caso universitario, los métodos activos que se están utilizando se asocian a menudo al constructivismo, corriente de pensamiento psicológica y epistemológica que posee múltiples variantes (Huber, 2008), por lo que es importante referirse a ella brevemente. Teóricos como J. Piaget, J. Brunner, L. Vygotsky, J. Dewey y E. von Glaserfeld son considerados como puntos de referencia obligados (Cooperstein \& KocevarWeidinger, 2004; Phillips, 1995). La premisa central de esta teoría del aprendizaje es que el conocimiento humano se adquiere a través de un proceso activo de construcción (Adams, 2006; Fox, 2001). En Piaget, por ejemplo, esta idea se relaciona con la noción de transformación:

En efecto, para conocer los objetos, el sujeto debe actuar sobre ellos y por consecuencia transformarlos. A partir de las acciones sensoriomotrices, los más elementales, hasta las operaciones intelectuales más refinadas que son aún acciones (reunir, ordenar, hacer corresponder, etc.), pero interiorizadas y ejecutadas en el pensamiento, elconocimiento estáconstantemente ligado a acciones o a operaciones, es decir, a transformaciones (Piaget, 1968, p.282).

Recordando la importancia d ada por Dewey a la acción del niño sobre su medio ambiente, el learning by doing encuentra así un fundamento importante en la psicología genética piagetiana (Piaget, 1969). Esto tiene también un aspecto histórico: Piaget fue presidente de la Oficina Internacional de Educación, cuyo fundador -Édouard Claparède- estuvo directamente ligado con la Educación Nueva, además de haber sido Adolphe Ferrière su director adjunto en un período. En efecto, Piaget habría sostenido los métodos activos a lo largo de toda su obra (Ducret, 2001). En palabras de Piaget:
"Sin importar el lazo que existe entre los principales innovadores pedagógicos, la psicología del niño y sus ideas pedagógicas maestras, es indudable que la gran corriente de la psicología genética moderna está en la fuente de los nuevos métodos" (Piaget, 1969, p. 212). Que hoy en día Piaget sea considerado como un referente del constructivismo aplicado a la Educación Superior y que no se enfatice este lazo histórico con la Escuela Nueva no deja de ser sorprendente.

Es importante considerar también la teoría sociocultural de Vygotsky, donde se establece que la construcción del conocimiento es un producto de la interacción, interpretación y de la comprensión social (Adams, 2006). Así, el estudiante aprende en un ambiente social (Huber, 2008), donde las interacciones estudiante-profesor y estudiante-estudiante son esenciales (Adams, 2006). Esto también es aplicable como verificación de la comprensión de los estudiantes de un cierto material (Cooperstein \& Kocevar-Weidinger, 2004). El profesor se transforma así en el garante de un ambiente de aprendizaje donde la experimentación y el diálogo son primordiales y surgen en torno a problemas o temas que son discutidos por los estudiantes y los profesores (Adams, 2006). Este elemento da un marco a los métodos que proponen la interacción entre los estudiantes, por ejemplo en el caso de métodos activos que promueven el trabajo en grupos, como el aprendizaje colaborativo, el aprendizaje basado en equipos, etc.

Otro elemento importante que se debe subrayar es el lugar que ocupa la experiencia en las teorías constructivistas. David Kolb ha edificado su teoría del aprendizaje experiencial, muy utilizada en la educación de adultos, a partir de las ideas de Dewey, Lewin y Piaget. Para Kolb, el aprendizaje es el proceso de creación de conocimiento a partir de la experiencia (Kolb, 1984; Svinicki \& Dixon, 1987).

Se han realizado varias investigaciones para evaluar el impacto de la utilización de las metodologías activas sobre el resultado del proceso de enseñanzaaprendizaje en el ámbito universitario (Costin, 1972). Evidentemente la heterogeneidad de métodos, la particularidad de la disciplina enseñada y los contextos donde estos métodos se aplican hacen que dicha evaluación sea delicada. Sin embargo, la evidencia 
empírica parece mostrar que la introducción de los métodos activos en los cursos magistrales tendría un impacto positivo en el aprendizaje de los estudiantes. Varios trabajos de compilación de investigaciones están disponibles. Por ejemplo, Bligh (1972) señala que la evidencia no muestra diferencia notable entre las clases magistrales y otros métodos, esto en el ámbito de la transmisión de la información. Sin embargo, esto cambia positivamente cuando con los métodos activos se trata de promover el pensamiento, el cambio de actitudes o el desarrollo de habilidades de comportamiento en los estudiantes. Por otra parte, y en el ámbito de la ingeniería, la compilación de Prince (2004) concluye que la introducción de actividades durante una clase magistral tendría un impacto positivo en el aprendizaje de los estudiantes.

El estudio más reciente que subraya la importancia de la implementación de los métodos activos fue publicado por Freeman y sus colegas (2014) en el ámbito de las ciencias, ingeniería y matemáticas. Los autores meta-analizaron 225 estudios al respecto, concluyendo que la utilización de estos métodos aumenta el desempeño en los tests estandarizados de conceptos científicos (concept inventories) y disminuye la proporción de estudiantes que reprueban un curso (Freeman et al., 2014).

Ejemplos importantes de tales métodos son el aprendizaje cooperativo/colaborativo, el método por proyectos, el aprendizaje basado en problemas, el aprendizaje basado en equipos, la instrucción por pares y el aula invertida. Por razones de espacio no profundizaremos en la descripción de estos métodos (la que puede ser encontrada en las referencias), y daremos solo ejemplos de instituciones que los han aplicado. De hecho, existen otros métodos, pero nos parece que los señalados dan una buena imagen de la aplicación de la pedagogía activa en el aula universitaria.

Respecto del aprendizaje cooperativo, su origen se puede encontrar en las ideas de Kurt Lewin (Sherman, 1991; Sherman, Schmuck \& Schmuck, 2006). Se trata aquí de establecer una dinámica de trabajo grupal en el aula basada en una serie de principios: a) la interdependencia positiva entre los miembros del grupo, b) la responsabilidad individual con relación al trabajo del grupo, c) la interacción entre sus miembros, d) la importancia de la heterogeneidad y el el desarrollo de habilidades sociales (Sherman et al., 2006, p. 196). Existe también la corriente colaborativa, que subraya la construcción social del conocimiento (constructivismo social), donde es fundamental la interacción de los individuos (Barkley, Major \& Cross, 2014). A pesar de las diferencias epistemológicas, a nivel práctico ambas palabras parecen ser utilizadas de manera equivalente y sus técnicas tienen en común el ser flexibles y adaptables en función de cada situación.

Otro método utilizado es el denominado método por proyectos. En este caso la relación con su equivalente en el ámbito escolar, proveniente de la Educación Progresista, es directo, ya que podemos encontrar su origen en un artículo publicado por Kilpatrick, discípulo de Dewey, en 1918 en el Teachers College Record. En este escrito considera el valor educativo de las actividades asociadas a los objetivos del alumno (Kilpatrick, 1929). Hoy en día este método sigue utilizándose a nivel primario y secundario (Larmer \& Mergendoller, 2010). Su aplicación en la enseñanza superior parece estar asociado al movimiento de protesta estudiantil de los años 60, en particular en el caso de Dinamarca y específicamente de las universidades de Roskilde (fundada en 1972) y de Aalborg (fundada en 1974). creadas por una comunidad de profesores muy influenciados por las ideas de Dewey y Piaget (Fayolle \& Verzat, 2009). Hoy en día el trabajo por proyectos continúa siendo un método pedagógico fundamental en esta universidad. Es importante subrayar la importancia de la multidisciplinareidad y de la elección de las problemáticas relacionadas con los intereses de los estudiantes, muchas veces ligados a problemáticas globales, lo que permite establecer un lazo entre la teoría y la práctica (Fayolle \& Verzat, 2009).

Similar a este último, el método del aprendizaje basado en problemas (PBL) permite diseñar e implementar un curso a partir de una situación-problema que funciona como fuente de motivación y de concentración para suscitar la participación activa de los estudiantes. Se trata de una manera de concebir el curriculum en torno a problemas de la práctica profesional, comenzando típicamente un curso PBL por la contextualización 
de un problema y no por el estudio de saberes de distintas disciplinas (Boud \& Feletti, 1991). Este método fue inicialmente aplicado en la enseñanza de la medicina, específicamente implementado en la Facultad de Ciencias de la Salud de la Universidad de McMasters, en Canada, en 1969 (Neville, 2009). El grupo de profesores que propusieron este método se inspiraron de la experiencia desarrollada en la Escuela de Derecho de la Universidad de Harvard en los años 20 (Schmidt, 1993). Entre otras universidades reputadas por sus dispositivos pedagógicos basados en esta línea se puede mencionar a la Universidad de Maastricht y la Universidad de Twente en los Países Bajos, a las universidades de Roskilde y Aalborg en Dinamarca, la Universidad de Newcastle en Australia y la de Bremen en Alemania (Du, De Graff \& Kolmos, 2009). Sin embargo, se encuentran hoy en día iniciativas inspiradas de estas experiencias en todo el mundo y en distintos campos como la medicina, la ingeniería, el trabajo social, la arquitectura, el derecho, la administración, por nombrar solo algunos.

Otro método relacionado con el trabajo grupal es el aprendizaje basado en equipos (TBL), el cual fue implementado por Larry Michelsen, profesor de administración, hacia fines de los años 70 en la Universidad de Oklahoma. Con este método se intenta dar a los estudiantes la oportunidad de practicar en clases, utilizando conceptos básicos para la solución de problemas (Michaelsen \& Sweet, 2008). Incluso si este se puede asociar al cooperativo, esta técnica ha desarrollado características y una metodología que le son propias, lo que hace que sea considerada como una metodología por sí misma (Michaelsen \& Fink, 2008). Esta ha sido utilizada en varias disciplinas: administración, salud, ingeniería, educación, etc.

En el ámbito del fomento de la actividad de los estudiantes, específicamente respondiendo al problema de aprovechar el tiempo de las clases como una instancia de aplicación del conocimiento y de interacción, más que como uno de trasmisión de conocimiento, debemos mencionar la sala de clases invertida o flipped classroom. En el contexto de la enseñanza superior, el artículo de Lage, Platt y Treglia (2000) sobre la experiencia con este método en la enseñanza de la economía parece ser uno de los pioneros. En sus palabras:

Invertir la sala de clases significa que las situaciones que antes tenían lugar fuera de la sala se desarrollan ahora en su interior y viceversa... Para esto la utilización de la World Wide Web y de los computadores multimedia permite a los estudiantes mirar las clases en un laboratorio de informática o en su casa, dejando las tareas y las actividades de aplicación en grupo para la clase (Lage, Platt \& Treglia, 2000, p.32).

La importancia del tiempo de clase destinado a los trabajos prácticos permite igualmente al profesor dar a los estudiantes retroalimentación regular sobre su trabajo (Warter-Perez \& Dong, 2012). Actualmente muchos profesores universitarios graban videos sobre los temas a tratar en sus clases, los dejan a disposición de sus estudiantes en Internet y orientan las clases hacia actividades de aplicación que clarifican el material visto por ellos. Desde el punto de vista de la pedagogía activa, esta estrategia permite involucrar al estudiante (a través de la actividad previa en línea) de la misma manera que fomenta la generación de un espacio de interacción entre estudiantes y de aplicación del conocimiento en clases.

\section{CONCLUSIONES}

A pesar de que los métodos activos se inscriben en una larga tradición, aparecen hoy en día como parte de la respuesta ante la problemática de la amplitud y de la calidad en la enseñanza universitaria, entendida esta última como la implementación de procesos de enseñanza-aprendizaje efectivos. Así, estos métodos son parte de una estructura que intenta enfatizar la importancia de la centralidad del aprendizaje de los estudiantes. Esto los ubica en un lugar que no es exactamente el mismo que el de sus antecesores de la Escuela Nueva y del Movimiento Progresista.

En efecto, como lo señalaba Ferrière, es necesario hacer una diferencia entre los métodos y la idea más amplia de la Educación Nueva y Progresista. Como hemos examinado, en estas últimas se trata de una filosofía de la educación que enfatiza la espontaneidad y el trabajo libre del estudiante, su desarrollo integral y -de manera más clara en el progresismo de Dewey- 
su desarrollo como futuro ciudadano en un sistema democrático. Las palabras de Piaget son aquí relevantes, trasponiéndolas a la Educación Superior:

La Escuela Nueva hace referencia a la actividad real, al trabajo espontáneo, basado en la necesidad y el interés personal [...] Ya que, debemos repetir con Dewey y Claparède, que el trabajo obligado es una anomalía antipsicológica y que toda actividad fecunda supone un interés (Piaget, 1969, p.222).

¿Es posible entender la Educación Superior con estas características? En relación con la espontaneidad y a la actividad libre, vemos que algunos de los métodos que se están utilizando, como por ejemplo el aprendizaje a través de problemas y el método de proyectos, dan espacio a la propia motivación del estudiante. Es quizás por eso que su implementación muchas veces no resulta fácil. Otros métodos, donde se busca mejorar los resultados de los estudiantes, sin atender especialmente a su espontaneidad o interés, parecen tener una función más bien instrumental.

Evitando el maniqueísmo, podemos decir que los métodos activos cumplen un rol práctico, donde el objetivo final es que los estudiantes aprendan en función de lo establecido por un conjunto de objetivos de aprendizaje. Son finalmente técnicas que permiten a los profesores universitarios -que por lo general no tienen una preparación pedagógica- desarrollar clases donde los estudiantes aumentan sus posibilidades de aprender. Es evidente que tratándose de la Educación Superior, existen aprendizajes específicos que los estudiantes deben desarrollar y que estos se articulan y toman sentido en relación con una descripción dada del profesional o graduado que se quiere formar. La pregunta, sin embargo, es si consideramos a ese conjunto de técnicas como parte integrante de una filosofía de la educación que enfatice la espontaneidad, la motivación y la libertad creativa del estudiante, como aspectos a desarrollar por los estudiantes universitarios.

En otro ámbito, y en relación con la integralidad de la formación de los estudiantes, el discurso de las habilidades blandas muestra hoy la importancia de esta idea. Pareciera que se entiende que la enseñanza universitaria, estando ad portas de la vida profesional de los estudiantes, tiene una responsabilidad importante en la formación de ciudadanos integrales. Sin embargo, no es claro que los métodos activos utilizados sean siempre explícitamente ligados a estas habilidades.

No basta tan solo formar buenos especialistas en áreas específicas sino también desarrollar habilidades que tocan los aspectos emocionales e interpersonales, fundamentales a la hora de insertarse en el mundo del trabajo. Desde este punto de vista vemos que los métodos activos en boga hoy en la universidad son una oportunidad para desarrollar estos aspectos. Elementos como la colaboración y la responsabilidad social aparecen como características que pueden ser trabajadas a partir de dichos métodos, en la medida que sean concebidos como parte de una filosofía de la educación más amplia. Desde ese punto de vista, la implementación de estos métodos en la universidad va en la dirección de la formación de ciudadanos para un mundo democrático, como lo soñaba Dewey. En este sentido es importante recordar que la Educación Nueva y la Progresista se desarrollaron en un ambiente de pre y posguerra, donde la formación de un nuevo ser humano era vista como la esperanza de un buen futuro para la humanidad.

En este sentido, y volviendo a la idea expuesta en la introducción de los elementos valiosos que podemos tomar de las experiencias de la Pedagogía Activa en el ámbito escolar, nos parece que es importante plantearse -siguiendo las observaciones de Ferrièreel paso de los métodos activos a la pedagogía activa. Esta última implica -de acuerdo con lo que hemos descrito anteriormente- lo que podríamos llamar una filosofía de la educación centrada radicalmente en el estudiante. Este paso nos parece estar enfocado en lo que discutiéramos al inicio de este apartado: la inclusión de la espontaneidad y de la propia motivación y libertad del estudiante en el proceso de enseñanza-aprendizaje, incluyendo la importancia de la multidimensionalidad e integralidad de su desarrollo. Desde un cierto punto de vista, se trata de desescolarizar la universidad y deshacer así el proceso de escolarización que ha sufrido a partir de la masificación de su acceso (Bourgin, 2011). En esta dirección, los estudiantes se transforman en más que actores, se vuelven co-constructores de su propio aprendizaje, definiendo por ejemplo objetivos de 
aprendizaje de asignaturas a la par que sus profesores y orientando su curso de estudios en la línea de sus propios intereses.

La misma tensión que vivieron los progresistas a nivel de la escuela la pueden vivir los profesores universitarios si es posible desarrollar la pedagogía activa en este ámbito, dando así espacio a que la creatividad y la espontaneidad de los estudiantes se manifieste. Es en este futuro desarrollo que los métodos pueden dar paso a la utopía, pasando de una lógica circunscrita a lo instrumental a otra que enfatice el ideal que es parte de la cadena de las ideas pedagógicas que nos viene del pasado.

\section{REFERENCIAS}

Adams, P. (2006). Exploring social constructivism: theories and practicalities. Education 3.13: International journal of primary, elementary and early years education, 34(3), 243-257. doi:10.1080/03004270600898893

Barkley, E., Major, C., \& Cross. K. (2014). Collaborative learning techniques. A handbook for college faculty. San Francisco: Jossey-Bass.

Barr, R., \& Tagg, J. (1995). From teaching to learning: a new paradigm for undergraduate education. Change: The Magazine of Higher Learning, 27(6), 13-25. doi:10.1080/00091383.1995.10544672

Béchard, J.-P. (2008). Fondements épistémologiques des auteurs clés de la pédagogie de l'enseignement supérieur : une analyse de trois revues 1976-2003. Revue des sciences de l'éducation, 34(3), 537-568. doi:10.7202/029508ar

Bligh, D. (1972). What's the use of lectures? San Francisco: Jossey-Bass. Blondeau, N., \& Couëdel, A. (2002). Une pédagogie critique à l'université. Pratiques de formation/analyses, 43, 27-39.

Bonwell, C., \& Eison, J. (1991). Active learning. Creating excitement in the classroom. ASHE-ERIC Higher Education Report No.1. Washington: The George Washington University, School of education and human development.

Boud, D., \& Feletti, G. (Eds.). (1991). The challenge of problem based learning. London: Kogan Page.

Bourgin, J. (2011). Les pratiques d'enseignement dans les universités de masse: cycles universitaires se scolarisent-ils? Sociologie du travail, 53(1), 93-108. doi:10.1016/j.soctra.2010.12.005

Chickering, A. W., \& Gamson, Z. F. (1987). Seven principles for good practice in undergraduate education. AAHE Bulletin, 39(7), 3-7.

Cooperstein, S., \& Kocevar-Weidinger, E. (2004). Beyond active learning: a constructivist approach to learning. References services review, 32(2), 141-148. doi:10.1108/00907320410537658

Costin, F. (1972). Lecturing versus other methods of teaching: a review of research. British journal of educational technology, 3(1), 4-31. doi:10.1111/j.1467-8535.1972.tb00570.x

De Ketele, J. M. (2010). La pédagogie universitaire: un courant en plein développement. Revue française de pédagogie, 172, 5-13.

Del Pozo Andrés, M. (2009a). El proceso de Bolonia en las aulas universitarias: una perspectiva europea. Cuestiones pedagógicas, 19, 55-73.

Del Pozo Andrés, M. (2009b). The transnational and national dimensions of pedagogical ideas: the case of the project method, 1918-1939. Paedagogica Historica, 45(4\&5), 561-584. doi:10.1080/00309230903100957

Deledalle, G. (1995). John Dewey. Paris : PUF.

Dewey, J. (1900). The school and society. Chicago: University of Chicago Press.

Dewey, J. (2003). Reconstruction en philosophie. Pau: Léo Scherer.

Du, X., De Graaff, E., \& Kolmos, A. (Eds.). (2009). Research on PBL practice in engineering education. Rotterdam: Sense Publishers.

Ducret, J-J. (2001). Constructivists : usages et perspectives en éducation. Perspectives, 31(2), 177-189.

Fayole,A., \&Verzat, C. (2009).Pédagogiesactivesetentrepreneuriat:quelle place dans nos enseignements? Revue de l'entrepreneuriat, $8(2), 1-15$.

Ferrière, A. (1911). Les écoles nouvelles. L'Angleterre et l'Allemagne. La Revue Illustré de Paris, 18, 611-631.

Ferrière, A. (2004). L'école active. Paris: Fabert.

Fox, R. (2001). Constructivism examined. Oxford Review of Education, 27 (1), 23-35. doi:10.1080/03054980125310

Freeman, S. et al. (2014). Active learning increases student performance in science, engineering, and mathematics. Psycological and cognitive sciences, 111(23), 8410-8415. doi:10.1073/ pnas. 1319030111

Hameline, D. (2004). Préface. En Ferrière, A. (Ed.), L'école active (pp. 7-29). Paris: Fabert.

Hameline, D. (2006). L'éducation nouvelle après l'éducation nouvelle. Paedagogica Historica, 42(1/2), 263-290.

Herold, J. (1974). Sputnik in American education: A history and reappraisal. McGill Journal of Education, 9(2), 143-164.

Huber, G. (2008). Aprendizaje activo y metodologías educativas. Revista de Educación - Ministerio de Educación de España: Tiempos de cambio universitario en Europa, 59-81.

Kilpatrick, W. (1929). The project method. The use of the purposeful act in the educative process. New York: Teachers College.

Kolb, D. (1984). Experiential Learning: Experience as the Source of Learning and Development. N.J: Prentice-Hall.

Lage, M. J., Platt, G. J., \& Treglia, M. (2000). Inverting the Classroom: 
A Gateway to Creating an Inclusive Learning Environment. The Journal of Economic Education, 31(1), 30-43. doi:10.1080/00220480009596759

Larmer, J. \& Mergendoller, J. (2010). 7 Essentials for project-based learning. Educational leadership, 68(1), 34-37.

Luzuriaga, L. (1944). La educación nueva. Buenos Aires: Losada.

McKeachie, W. (1990). Research on college teaching: the historical background. Journal of educational psychology, 82(2), 189-200. doi:10.1037/0022-0663.82.2.189

McKeachie, W. J., Pintrich, P. R., Lin, Y. G., \& Smith, D. A. (1987). Teaching and learning in the college classroom: A review of the literature. Ann Harbor, National Center for Research to Improve Postsecondary Teaching and Learning: The University of Michigan.

Michelsen, L., \& Fink, L. (2008). Preface. New directions for teaching and learning, 116, 1-5. doi:10.1002/tl.329

Michelsen, L., \& Sweet, M. (2008). The essential elements of teambased learning. New directions for teaching and learning, 116, 7-27. doi:10.1002/tl.330

Miller, R. (2002). Free schools, free people. NY: SUNY.

National Institute of Education. (1984). Involvement in learning: Realizing the potential of American higher education. Washington D.C.:U.S. Department of Education.

Neville, A. (2009). Problem-based learning and medical education forty years on. Medical Principles and Practice, 18, 1-9. doi: $10.1159 / 000163038$

Ohayon, A., Ottavi, D. \& Savoye, A. (2004). L'éducation nouvelle, histoire, présence et devenir. Berne: Peter Lang.

Oelkers, J. (2006). Raformpädagogikvor Reformpädagogik. Paedagogica Historica, 42(1\&2), 15-48.

Padilla, T. \& Gil, J. (2008). La evaluación orientada al aprendizaje en la Educación Superior: condiciones y estrategias para su aplicación en la docencia universitaria. Revista Española de Pedagogía, 66(241), 467-486.

Phillips, D. C. (1995). The Good, the Bad and the Ugly: The many faces of constructivism. Educational researcher, 24(7), 5-12. doi: $10.2307 / 1177059$

Piaget, J. (1968). Le point de vue de Piaget. Journal international de psychologie, 3(4), 281-299.

Piaget, J. (1969). Psychologie et pédagogie. Paris: Denoel-Gonthier
Prince, M. (2004). Does active learning work? A review of the research. Journal of engineering education, 93(3), 223-231. doi: 10.1002/ j.2168-9830.2004.tb00809.x

Resweber, J. P. (1986). Les pedagogies nouvelles. Paris: PUF.

Reynolds, K. (1997). Progressive ideals and experimental higher education: The example of John Dewey and Black Mountain College. Education and Culture, 14(1), 1-9.

Rogers, C., \& Freiberg, H. J. (1994). Freedom to learn. New York: Macmillan.

Schmidt, H. (1993). Foundations of problem based learning. Some explanatory notes. Medical education, 27, 422-432. doi:10.1111/j.1365-2923.1993.tb00296.x

Sherman, L. (1991). Cooperative learning in post secondary education: implications from social psychology for active learning experiences. Paper presented at the annual meeting of the American Education Research Association (Chicago, April 3-7 1991).

Sherman, L. W., Schmuck, R., \& Schmuck, P. (2006). Kurt Lewin's contribution to the theory and practice of education in the United States: The importance of cooperative learning. In J. Trempala, A. Pepitone \& B. H. Raven (Eds.) Lewinian Psychology. Procedings of the International Conference Kurt Lewin: Contribution "to contemporary psychology" (pp. 191207). Bydgoszcz, Poland: Kazimier Wielki University Press.

Svinicki, M. \& Dixon, N. (1987). The Kolb Model modified for classroom activities. College Teaching, 35(4), 141-146.

Townsend, B., Jackson, L., \& Wiese, M. (1992). Creating distinctiveness: lessons from uncommon colleges and universities. ASHEERIC Higher Education Report No. 6. Washington, D.C.: The George Washington University, School of education and human development.

Vasquez, A. \& Oury, F. (1967). Vers une pédagogie institutionnelle. Paris: Maspero.

Warter-Perez, N. \& Dong, J. (2012). Flipping the classroom: how to embed inquiry and design projects into a digital engineering lecture. Proceedings of the 2012 ASEE PSW Section Conference.

Zilversmit, A. (1993). Changing schools. Progressive education theory and practice, 1930-1960. Chicago: The University of Chicago Press. 